\title{
Thermal Vibrational Convection in a Two-phase Stratified Liquid
}

\author{
Qingming CHANG \& J. Iwan D. ALEXANDER
}

National Center for Space Exploration Research and Department of Mechanical and Aerospace Engineering, Case Western Reserve University, 10900 Euclid Ave., Cleveland, Ohio 44106, U.S.A.

Contact :

Tél : 216368 6045. Télécopie : 2163686445.

\section{Convection thermo - vibrationnelle d'un système liquide à deux phases et stratifié}

Résumé : La réponse d'un système liquide à deux pahses et stratifié sujet à une vibration parallèle à un gradient de température imposé est analysée en utilisant une méthode hybride thermique LatticeBoltzmann (HTLB). Les vibrations considérées correspondent aux translations sinusoïdales d'une cavité rigide à une fréquence fixe. Les couches sont couplées thermiquement et mécaniquement. L'interaction entre la convection thermique causée par la gravité et les vibrations est étudiée. La capacité des vibrations à intensifier l'écoulement, le transfert de chaleur et la déformation de l'interface est étudié. Pour la gamme des paramètres considérés, les résultats indiquent que l'effet du nombre vibratoire de Rayleigh et de la fréquence vibratoire sur un système liquide à deux phases et stratifié est très différent que son effet sur un système liquide à une phase. La compraraison de ces deux systèmes et discutée.

\section{Mécanique des fluides numérique / convection thermique-vibratoire / lattice-Boltzmann / interface}

\begin{abstract}
The response of a two-phase stratified liquid system subject to a vibration parallel to an imposed temperature gradient is analyzed using a hybrid thermal lattice Boltzmann method (HTLB). The vibrations considered correspond to sinusoidal translations of a rigid cavity at a fixed frequency. The layers are thermally and mechanically coupled. Interaction between gravity-induced and vibration-induced thermal convection is studied. The ability of applied vibration to enhance the flow, heat transfer and interface distortion is investigated. For the range of conditions investigated, the results reveal that the effect of vibrational Rayleigh number and vibrational frequency on a two-phase stratified fluid system is much different than that for a single-phase fluid system. Comparisons of the response of a two-phase stratified fluid system with a singlephase fluid system are discussed.
\end{abstract}

Computational fluid dynamics/ thermal vibrational convection / lattice-Boltzmann / interface

\section{Introduction}

Thermal convection of a single-phase fluid system in an enclosure induced simultaneously by gravity and vibration has received a great deal of attention in the past because of its importance in material processing or in heat transfer under vibrational environment [1-4] The influence of various factors, such as vibrational direction, vibrational Rayleigh number and frequency etc. on structure of the convective flows and the heat transfer in the cavity have been investigated. Particular attention has been given to mean or thermovibrational convection of single component fluids in plane fluid layers and cylindrical cavities, and includes the effects of gravity or weightlessness (See Ref.1 and references therein.) 
Studies of thermal convection in a two-layer fluid system with external vibration are important for evaluating the effect of the unavoidable vibrations of satellites and spacecraft mechanical structures on twophase fluid systems. The investigation of the behavior of two-layer fluid systems subject to vibration has mostly dealt with isothermal systems. Kenner [5] discussed theoretically the stability of the interface separating two immiscible incompressible fluids of different densities and viscosities in the case of fluids filling a cavity which performs horizontal harmonic oscillations. Duval et al. [6] focused on the interfacial dynamics of two-layer liquids under an oscillating gravitational field under isothermal conditions. They found that the interfacial region acts as a vortex source sheet and is susceptible to Kelvin-Helmholtz and Rayleigh-Taylor instabilities. The vortices produced along the interface can serve as a stirring mechanism to promote local mixing. Ivanova et al. [7] examined interfaces between immiscible fluids under horizontal vibration.

In this paper, results of a set of simulations of vibrational convection in a non-isothermal two-layer fluid system are reported. The effects of external high-frequency vibration on not only the flow characteristics and interfacial dynamics, but also the heat transfer process are examined.

\section{Numerical Model}

The physical model is shown in Fig. 1. A rigid cavity containing two different immiscible liquids and subject to a periodic lateral translation, $b \cos (\omega t) n$ with fixed amplitude and frequency is examined. Here $b$ is the displacement amplitude, $\boldsymbol{n}$ is a unit vector parallel to the translation direction and $\omega$ is the angular frequency of vibration. Fluid motion in the container is modeled using the two-phase thermal lattice Boltzmann model (TLBM) described in Refs. 8, and 9 with a straightforward modification to account for motion of the cavity. Additional details concerning modeling two-phase fluids using the lattice Boltzmann method are found in Ref. 10. In the TLBM the fluid motion is computed using a two-particle distribution function approach proposed by $\mathrm{He}$ et al. [11, 12]. The two distribution functions satisfy the following equations for distribution functions $\xi(\boldsymbol{r}, \boldsymbol{e}, t)$ and $f^{\phi}(\boldsymbol{r}, \boldsymbol{e}, t)$ where $\boldsymbol{e}$ is a so-called microscopic velocity [8$10,11], \boldsymbol{r}$ is the position and $t$ is time.

The Boltzmann equation for $\xi(\boldsymbol{r}, \boldsymbol{e}, t)$ is [8-10]

$$
\begin{aligned}
& \partial_{t} \xi(\boldsymbol{r}, \boldsymbol{e}, t)+\boldsymbol{e} \cdot \nabla_{\boldsymbol{r}} \xi(\boldsymbol{r}, \boldsymbol{e}, t) \\
& =-\frac{\xi-\xi^{e q}}{\tau(\varphi)}+(\boldsymbol{e}-\boldsymbol{u}) \cdot\left[\left(\boldsymbol{F}_{s}+\boldsymbol{G}\right) \Gamma(\boldsymbol{u})+\nabla \psi(\rho)(\Gamma(\boldsymbol{0})-\Gamma(\boldsymbol{u}))\right],
\end{aligned}
$$

where $\boldsymbol{G}(t)$ is a body force and

$$
\xi^{e q}=R T_{0} f^{e q}+\psi(\rho) \Gamma(\boldsymbol{0}), \Gamma(\boldsymbol{u})=\frac{1}{\left(2 \pi R T_{0}\right)^{9 / 2}} \exp \left[-\frac{(\boldsymbol{e}-\boldsymbol{u})^{2}}{2 R T_{0}}\right]
$$

The term $\boldsymbol{F}_{s}=\kappa \rho \nabla \nabla^{2} \rho$ represents the macroscopic interfacial force between the phases which is associated with a steep density gradient. Bulk molecular interactions are accounted for through the potential $\psi=B R T_{0} \rho^{2} \chi-A \rho^{2}$, and the parameters $A$ and $B$ can be set so as to determine the degree of phase separation. For fixed values of $A$ and $B$ the parameter $\kappa$ determines the magnitude of the interfacial tension. The pressure and momentum are computed from distribution function $\xi(\boldsymbol{r}, \boldsymbol{e}, t)$ as follows:

$$
p=\int \xi d \boldsymbol{e},
$$




$$
\rho \boldsymbol{u}=\frac{1}{R T_{0}} \int \xi \boldsymbol{e} d \boldsymbol{e}
$$

where the integral is over the microscopic velocity space. To calculate the density it is convenient to define an index function $\phi$ (which represents, in some sense the phase fraction) such that

$$
\phi=\int f^{\phi} d \boldsymbol{e} .
$$

The distribution function, $f^{\phi}=f^{\phi}(\boldsymbol{r}, \boldsymbol{e}, t)$, for the index function $\phi$ must be calculated as a function of time and position. This will be discussed below. The density is then calculated from the index function and is

$$
\rho(\phi)=\rho_{l}+\frac{\phi-\phi_{l}}{\phi_{h}-\phi_{l}}\left(\rho_{h}-\rho_{l}\right) .
$$

Here a subscript $l$ and $h$ denote the light and heavy fluid, respectively. Instead of setting transition values of the density $\rho$ at $T_{0}=0.883 T_{c r}$, the transition values of the index function $\phi$ are set to be $\phi_{l}=0.0403$ and $\phi_{h}=0.2589$, respectively, for a given practical situation the proper density values are then determined by (6) after setting $\rho_{h}$ and $\rho_{l}$ to the appropriate values. The dependence of density on temperature is introduced through $\rho_{h}=\rho_{h}(T)$, and $\rho_{l}=\rho_{l}(T)$. The distribution function, $f^{\phi}$, for the index function $\phi$ satisfies [12]

$$
\partial_{t} f^{\phi}(\boldsymbol{r}, \boldsymbol{e}, t)+\boldsymbol{e} \cdot \nabla_{\boldsymbol{r}} f^{\phi}(\boldsymbol{r}, \boldsymbol{e}, t)=-\frac{f^{\phi}-f^{\phi e q}}{\tau(\varphi)}-\frac{\nabla \psi(\phi) \cdot(\boldsymbol{e}-\boldsymbol{u})}{\phi R T_{0}} f^{\phi e q},
$$

where

$$
f^{\phi e q}=\frac{\phi}{\left(2 \pi R T_{0}\right)^{\text {/2 }}} \exp \left[-\frac{(\boldsymbol{e}-\boldsymbol{u})^{2}}{2 R T_{0}}\right] .
$$

In summary, to simulate Boussinesq-type incompressible multiphase flow, two distribution functions, $\xi$, $f^{\phi}$ are employed. The index distribution function $f^{\phi}$ is used to track the density distribution through equations (7), (5) and (6), while the auxiliary distribution function $\xi$ is used to obtain the pressure and momentum fields through equations (3) and (4). The lattice Boltzmann equations are discretized and are coupled with a macroscopic energy equation that provides an updated temperature field which is used to calculate the density in the discretized body force term $\boldsymbol{G}(t)$.

It is convenient to describe the fluid motion in a frame of reference that moves with the translational displacement of the cavity. Thus, the fluid velocity, relative to this moving frame, will be zero at cavity boundaries. As a consequence of this change of reference frame oscillatory translations of the container appear as a time-dependent acceleration $\boldsymbol{g}(t)=\boldsymbol{g}_{0}-b \omega^{2} \cos (\omega t) \boldsymbol{n}$, where $\boldsymbol{g}_{0}$ denotes the steady acceleration due to gravity at the earths surface. In the hybrid thermal lattice Boltzmann model (TLBM) $\boldsymbol{g}(t)$ appears in a body force term $\boldsymbol{G}(t)=\rho(\varphi) \boldsymbol{g}(t)$ in eq. (1).

The aspect ratio, $A r$, of the cavity is set to be $A r=2$ unless otherwise stated. The upper layer fluid II (silicone oil, 2cSt) is assumed to be a density-linear fluid while bottom layer fluid I (water) a densityinversion fluid with a quadratic dependence of density on temperature. Each fluid is characterized by its thickness $h_{i}$, thermal conductivity $k_{i}$, specific heat at constant volume $c_{v i}$, volumetric mass density $\rho_{i}$, 
kinematic viscosity $v_{i}$, and thermal expansion coefficient $\beta_{i}$. Unless otherwise mentioned, the ratio of the physical properties of the fluid II to fluid I have been fixed as $\rho_{r}=0.9, k_{r}=0.19, c_{v r}=0.4, v_{r}=1.27$. Initially, two layers of immiscible fluids at equal heights $\left(h_{1}=h_{2}=0.5 \mathrm{H}\right)$ fill the cavity and are at rest and heat transfer is by conduction (a linear horizontal temperature gradient in $x$ ). The dimensionless variables for this problem are the Rayleigh numbers, $R a_{1}=g \lambda_{1} \Delta T^{2} L^{3} / \alpha_{1} v_{1}, R a_{2}=g \beta_{2} \Delta T^{2} L^{3} / \alpha_{2} v_{2}$ (here $\Delta T=T_{h}-T_{c}$, where $T_{h}$ and $T_{c}$ are, respectively, the hot and cold temperatures of the vertical walls), $\beta_{2}=\frac{1}{\rho_{2 r}} \frac{d \rho_{2}}{d T}$ and $\lambda_{1}$ are related to the thermal expansion coefficient in the lower layer through $\beta_{1}=\lambda_{1}\left(T-T_{r e f}\right)$. The Prandtl number is $\operatorname{Pr}=v_{2} / \alpha_{2}$, and the capillary number is $\mathrm{Ca}=\rho_{2} v_{2} \alpha_{2} / \sigma_{0} L$, The Prandtl number is fixed at $\operatorname{Pr}=27.6$. Another important dimensionless parameter is the density inversion parameter that describes the location of the density inversion temperature with respect to the sidewall temperatures. It is given as

$$
I=\frac{T_{i n v}-T_{c}}{T_{h}-T_{c}}=\theta_{i n v} .
$$

Note that, the dimensionless temperature $\theta$ lies in the interval $0 \leq \theta \leq 1$ while $-\infty \leq I \leq+\infty$.

Two additional dimensionless parameters are necessary to characterize the vibration conditions. They are the vibrational Rayleigh number and dimensionless frequency and are given by $R a v=\left(\beta_{2} b \omega \Delta T L\right)^{2} / 2 v_{2} \alpha_{2}$ and $\Omega=\omega L^{2} / \alpha_{2}$, respectively. The effect of translational vibration on two-phase fluid flow is examined for fixed $R a_{1}=160, R a_{2}=5 \times 10^{3}, C a=3.2 \times 10^{-3}, I=0.43$ (thermocapillary forces are neglected).

The local Nusselt number and the average value at vertical walls are

$$
\begin{gathered}
N u=\frac{L}{\Delta T}\left(\frac{\partial T}{\partial x}\right)_{\text {wall }}=\left(\frac{\partial \theta}{\partial X}\right)_{X=0,1}, \\
\bar{N} u=\frac{1}{H \times W} \int_{0}^{H} \int_{0}^{W} N u \cdot d z d y=\frac{1}{H} \int_{0}^{H} N u \cdot d y=\frac{1}{A r} \int_{0}^{A r} N u \cdot d Y .
\end{gathered}
$$

The Rayleigh numbers are kept constant at $R a_{1}=160, R a_{2}=5 \times 10^{3}$, and $C a=3.2 \times 10^{-3}$, and $I=0.43$.

We first calculated thermal convection in both layers due to gravity alone and then examined the effects of vibration for a range of vibration amplitudes and frequencies.

\section{Results and Discussion}

\subsection{Convection due to gravity alone}

In the upper density-linear fluid layer, because the density gradient is negative, $d \rho / d T<0$, the fluid with lower density rises near the hot sidewall while that with higher density descends near the cold sidewall, which produces a clockwise convective roll (also called "regular roll"). However, in the lower layer, $I=0.43$ divides the fluid into two sections, the left and right sections. In the left section, the temperature is higher than the density inversion point and "regular roll" cell shown in Fig. 2(a) develops. In the right section, the temperature is lower than density inversion temperature and a second anticlockwise convective roll (so called "inversion roll") cell forms. However, this flow pattern is different with that in the singlephase fluid with density inversion where both rolls extend from bottom to top of the liquid layer and can be symmetric [17]. Due to the strong viscous coupling between the top density-linear fluid layer and bottom density-inversion fluid layer, the convection in the top layer enhances the "inversion roll" cell of the right section and squeezes and pushes the "regular roll" cell in the left section to the left-bottom corner. A density inversion plane can be assumed between these two sections where $I=0.43$ as shown in Fig. 2(a). The 
temperature profiles can also reflect the effect of different vorticity convection rolls. As show in Fig. 2 (c), the temperature distribution in the upper layer distort seriously because a strong clockwise convection.

\subsection{Convection induced by both gravity and vibration}

Vibration in a direction parallel to the temperature gradient imposed on the two-layer fluid system produces the response shown in Fig. 3. The vibrational Rayleigh number and frequency for this case are Rav $=5000$ and $\Omega=500$, respectively. Results are presented in terms of dimensionless variables. The quantities $L$, $L^{2} / \alpha_{2}, \Delta T=T_{h}-T_{c}$ are taken to scale length, time and temperature, respectively. For a high frequency vibration, the velocity and temperature always oscillate with a single dominant frequency as shown in Figure 3 and the phase difference with the driving force is either close to $\pi$ or $\pi / 2$, for velocity or temperature, respectively. Figure 4 displays the time histories of the average Nusselt number at the vertical cold wall. In general, the average Nusselt number increases from zero to a steadily oscillating periodic value.

Figure 5 (a) - (g) shows the streamlines at different times in one oscillation period after the flow field reaches a steady oscillation state. The periodic flow is established quickly after about 100 periods. Within contrast to the case of fluid flow without vibration (see Figure 2), only one oscillating convective roll is generated in the entire two-layer system, as shown in Figure 5 (a) - (g). The direction and intensity of the convection depend on time. To further study the effect of vibration on fluid flow, it is useful to examine the time average values of some variables, such as velocity, temperature etc.. The mean value of the velocity is

$$
\overline{\boldsymbol{u}}=f_{r} \int_{t}^{t+1 / f_{r}} \boldsymbol{u} d t
$$

where $f_{r}$ is vibrational frequency $f_{r}=\Omega / 2 \pi$, and the mean stream function contours are derived from the time averaged velocity field at steady oscillation state.

The mean stream function at the steady oscillation state is established and shown in Fig. 5 (h). Compared with that of fluid flow without vibration as shown in Fig. 2 (a), the roll cell located at the left-bottom corner moves up to the position near the interface while another anticlockwise roll cell is generated above it. Figure 6 (a) - (c) shows evolution of the associated temperature field. The distortion of the isotherms during the oscillation is small, but measurable. The mean temperature contours are plotted in Fig. 6 (d). The isotherms have maximum distortion near the top of the cavity and straighten out near the interface. Compared with Figure 2 (c), the effect of vibration is that the mean temperature field has evolved toward a conduction-like profile. This observation is consistent with the results in [1] and [4].

\subsection{Effect of vibrational Rayleigh number Rav}

Figures 7 and 8 show the mean streamlines and isotherms at a fixed vibrational frequency $\Omega=500$ for different vibrational Rayleigh numbers Rav. In Fig.7, two convective rolls with different senses of rotation near the interface and the left boundary wall grow with increased vibrational Rayleigh number Rav while the other two convective rolls are suppressed. The magnitude of the time-averaged velocity vector increases with larger values of Rav. A measure of the time-averaged velocity $U_{\text {avg }}$ is the average value of the mean velocity over all the nodes in the whole system

$$
U_{\text {avg }}=\frac{1}{n} \sum_{i}^{n} \sqrt{\bar{u}_{x}^{2}+\bar{u}_{y}^{2}} .
$$

Here $n$ is the total grid number, $\bar{u}_{x}, \bar{u}_{y}$ are the components of mean velocity vector $\overline{\boldsymbol{u}}$. The average velocity $U_{\text {avg }}$ increases with increased vibrational Rayleigh number Rav at any constant frequency $\Omega$, although the 
rate is very small at low frequency $(\sim \Omega=200$ ). The increased average velocity induced by vibration enhances heat transfer, and results in an increased Nusselt number $\bar{N} u$ (see Fig. 9).

The instantaneous location of the interface also oscillates with the external vibrations, and has the appearance of a traveling wave. Larger values of the vibrational Rayleigh number Rav accentuate the distortion of the interface, as shown in Figure 10. When the vibrational Rayleigh number Rav is large enough, the interface can reach the top and/or bottom of cavity, and the two fluids start to mix, further investigation of this is beyond the scope of this paper.

Previous studies have predicted that the thermal vibrational fluid flows of single-phase systems are very different from the behavior exhibited above. For a single-phase system with a vibration direction parallel to temperature gradient, increasing $R a v$ will generally decrease the mean velocity and the heat transfer rate (Nusselt number). This is because the vibration acts as an effective gravity force that tries to reorient isopycnic surfaces to be perpendicular to the vibration direction [4]. The mechanism for this difference is very interesting and is discussed below.

For a thermal vibrational convection in an enclosure filled with single-phase fluid system under weightlessness ( $g=0, R a=0$ ), no mean motion in the fluid is expected when the vibration direction is perpendicular to the isotherms for high frequency vibration [4], since there is no pre-existing gravityinduced buoyancy flow. The situation is analogous to the unidirectional oscillatory translation of a simply supported rigid pendulum in a direction parallel to its length under zero gravity conditions. However, in a two-layer fluid system at the same conditions as above, the vibration-induced phase-buoyancy force causes the entire system to oscillate. This is simply because the interface separating the two different density phases is initially flat and parallel to the vibration direction; the density gradient across the interface is perpendicular to the vibration direction. Thus, the interface is an isopycnic surface and will tend to rotate to be perpendicular to the vibration direction. This is in contrast to thermally induced density variations within each phase for which the local density gradient is parallel to the vibration direction. As the direction of translation (corresponding to the vibration) of the cavity changes from left to right, the interface will change shape or sloshes back and forth, sustaining an oscillatory motion of the fluid. This flow will not occur for a similarly oriented single-phase fluid. Thus, in the single-phase case studied by Zhao [4] with vibration parallel to the applied thermal gradient the mean flow approaches zero as the vibrational Rayleigh number Rav increases at a constant frequency $\Omega$. In contrast, for the mean flow velocity in the two-phase case with vibration parallel to the applied thermal gradient, the mean velocity does not approach zero but in fact increases with increasing values of $R a v$. As a result, the heat transfer rate $\bar{N} u$ is enhanced by the vibration.

Convection in this two-layer fluid system arises due to two sources (see Fig. 11); one is natural convection due to steady gravity oriented perpendicular to the applied temperature gradient, the second is vibrationinduced convection which for this two-phase situation involves thermovibrational buoyancy within each layer and interfacial buoyancy due to the density difference between the phases. The mean flow in a twolayer system is enhanced by increasing the vibrational Rayleigh number Rav due to buoyant flow caused by the sloshing of the interface. When Rav is large enough, the mean flow induced by vibration will dominate the fluid flow and heat transfer in the two-layer fluid system. That is why, for the particular geometry studied here, a lager value of vibrational Rayleigh number intensifies the fluid flow in two-layer fluid system while suppresses flow in single-phase system.

\subsection{Effect of vibrational frequency $\Omega$}

Figure 9 reveals the effect of vibrational frequency on the thermovibrational dynamics of a two-layer system on heat transfer rates. For the range of frequencies studied here $200<\Omega<1000$, and at a fixed vibrational Rayleigh number Rav the mean flow velocity continues to be enhanced as the frequency is increased. Hence, the heat transfer rate $\bar{N} u$ is increased even though the temporal and mean distortion of the interface is suppressed. This is because the rapid small amplitude interfacial oscillations generate a strong mean flow. 
The interface is a vibrational buoyancy source activated by the vibration that is roughly parallel to the interface. In contrast, in a single-phase fluid system, the absence of the interface allows the isotherms to become almost parallel to the vertical walls. In this case, the mean flow velocity and heat transfer rate are independent of the vibrational frequency $\Omega$ [4].

\section{Conclusions}

The effect of external vibration on the convective flow and heat transfer in a two-layer fluid system of immiscible liquids with density inversion was investigated using a hybrid thermal lattice Boltzmann method. Under the external vibration corresponding to an oscillatory uniform horizontal translation of the rigid cavity, oscillatory convection is established after a steady oscillation state is reached. For the range of conditions investigated, the results reveal that the effect of vibrational Rayleigh number Rav and vibrational frequency $\Omega$ on a two-layer fluid system is much different than that for a single-phase fluid system when the vibration direction is parallel to the temperature gradient. In a two- layer system consisting of immiscible liquids with a density inversion, the mean flow velocities and heat transfer rate increase as Rav and $\Omega$ increase. This is because the mean fluid motion in a two-layer fluid system subject to translational vibration is not zero even under weightlessness. This is in contrast to a single-phase fluid system, where the natural convection is suppressed and the Nusselt number decreases and tends towards unity as $\operatorname{Rav}$ and $\Omega$ are increased. The interface between two-layer fluids experiences larger distortion at larger values of Rav and smaller values of $\Omega$.

The effect of Rav and $\Omega$ on a two-layer fluid system is much different than for a single-phase fluid system when the vibration direction is parallel to the temperature gradient. In a two-layer system consisting of immiscible liquids with a density inversion, the mean flow velocities and heat transfer rate increase as Rav and $\Omega$ increase. This is because the mean fluid motion in a two-layer fluid system subject to translational vibration is not zero even under weightlessness. This is in contrast to a single-phase fluid system, where the natural convection is suppressed and the Nusselt number decreases and tends towards unity as Rav is increased. The interface between two-layer fluids experiences larger distortion at larger values of Rav and smaller values of $\Omega$.

\section{Acknowledgements}

The authors acknowledge support from the National Aeronautics and Space Administration through NASA grant NAG8-1727 and through the National Center for Space Exploration Research.

\section{References}

1. Gershuni G.Z. , Lyubimov, D.V., Thermovibrational Convection, Wiley, 1998.

2. Simonenko I.B., Foundation of average method for the problem of convection in the field of rapidly oscillating forces and for other parabolic equations Math. Sb., 87[129] (1972)236.

3. Khallouf H., Gershuni G.Z., Mojtabi A., Numerical study of two-dimensional thermovibrational convection in rectangular cavities, Numerical Heat Transfer, A, 27 (1995)297.

4. Zhao Y., "Numerical Analysis of Thermal and Thermosolutal Vibrational Convection and its Effect on Heat and Mass Transport”, Ph.D. dissertation Case Western Reserve University, 2002.

5. KhennerM.V., Lyubimov D.V., Belozerova T.S., Roux B., Stability of plane-parallel vibrational flow in a twolayer system, J. Mech. B/Fluid 18 (1999) 1085.

6. Duval W.M.B., Jacqmin D.A., Interfacial dynamics of two liquids under an oscillating gravitational field, AIAA Journal, 28 (1990) 1933.

7. Ivanova A. A., Kozlov V. G., Evesque, P., Interface dynamics of immiscible fluids under horizontal vibration, Fluid Dynamics 36(3) (2001) 362.

8. Chang Q., Alexander J.I.D., Application of the lattice Boltzmann method to two-phase Rayleigh-Benard convection with a deformable interface. J. Comp. Phys., 212 473-489 (2005).

9. Chang Q., Ph.D. Lattice Boltzmann Method (LBM) for Thermal Multiphase Fluid Dynamics, PH.D. Dissertation Case Western Reserve University 2005. 
10. Chang Q., Alexander J.I.D., Analysis of single droplet dynamics on striped surface domains using a lattice Boltzmann method. Microfluidics and Nanofluidics, Issue: Online first pp. 1-18 http://dx.doi.org/10.1007/s10404005-0075-2 (2006).

11. He X., Doolen G., 2002, Thermodynamic foundations of kinetic theory and lattice Boltzmann models for multiphase flows, J. Stat. Phys., 107(1/2), 309.

12. He X., Chen S., Doolen G., A novel thermal model for the lattice Boltzmann method in incompressible limit, J. Comput. Phys., 146 (1998) 282. 


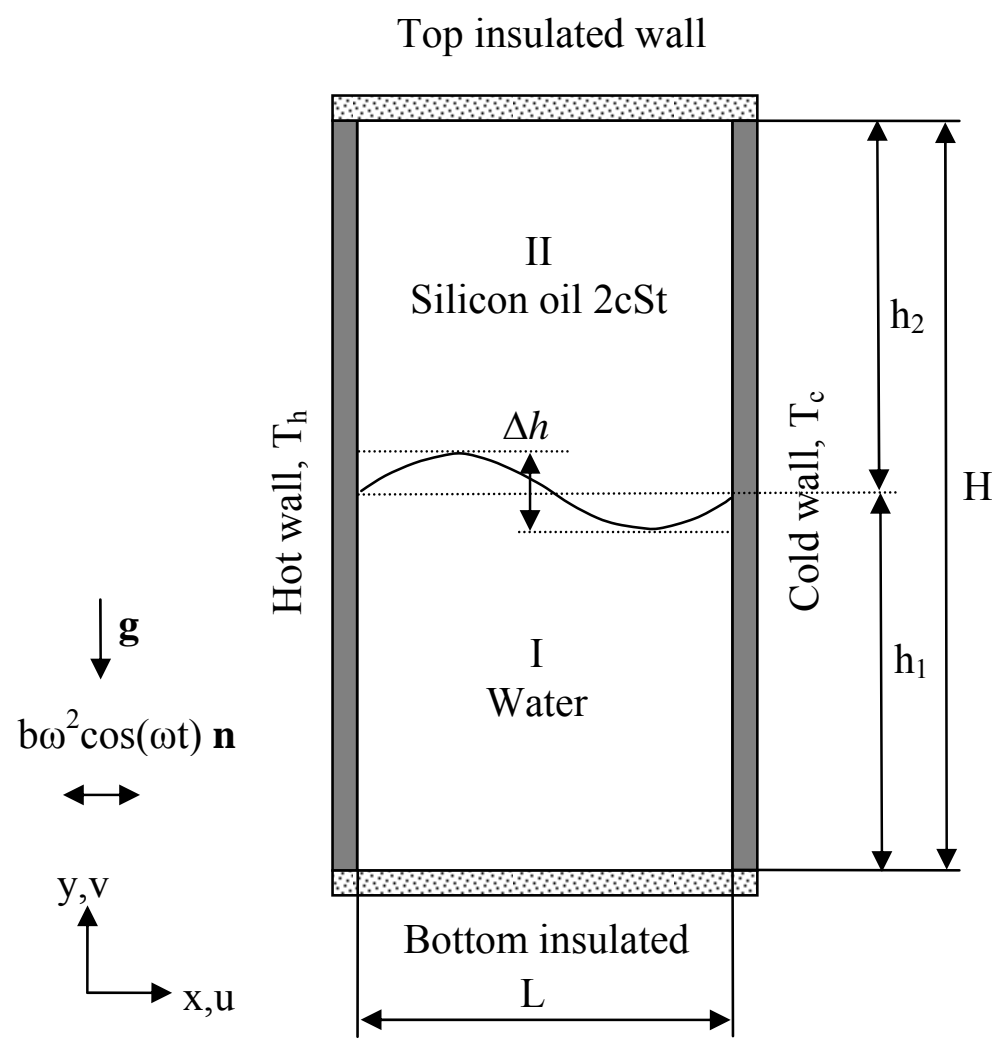

Fig. 1 Schematic of a two-layer system heated from the side walls.

Fig.1 Système à deux couches chauffé latéralement. 


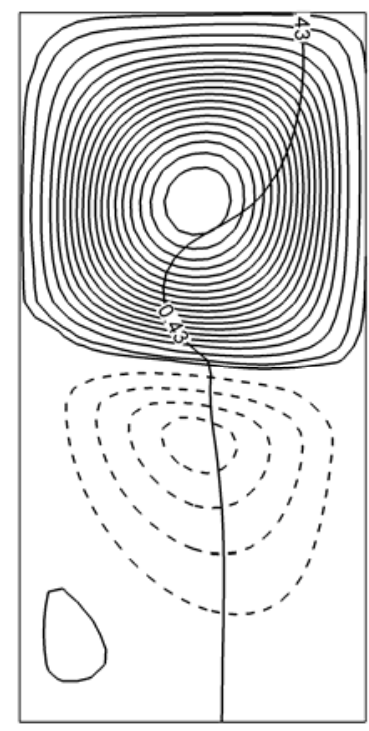

(a) streamlines

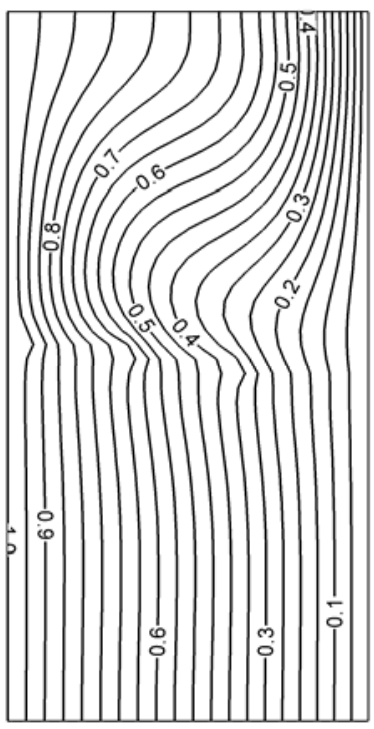

(b) isotherms

Fig. 2 Buoyant thermal convection with $R a v=0$.

Fig. 2 Convection thermique pour $\operatorname{Rav}=0$. 


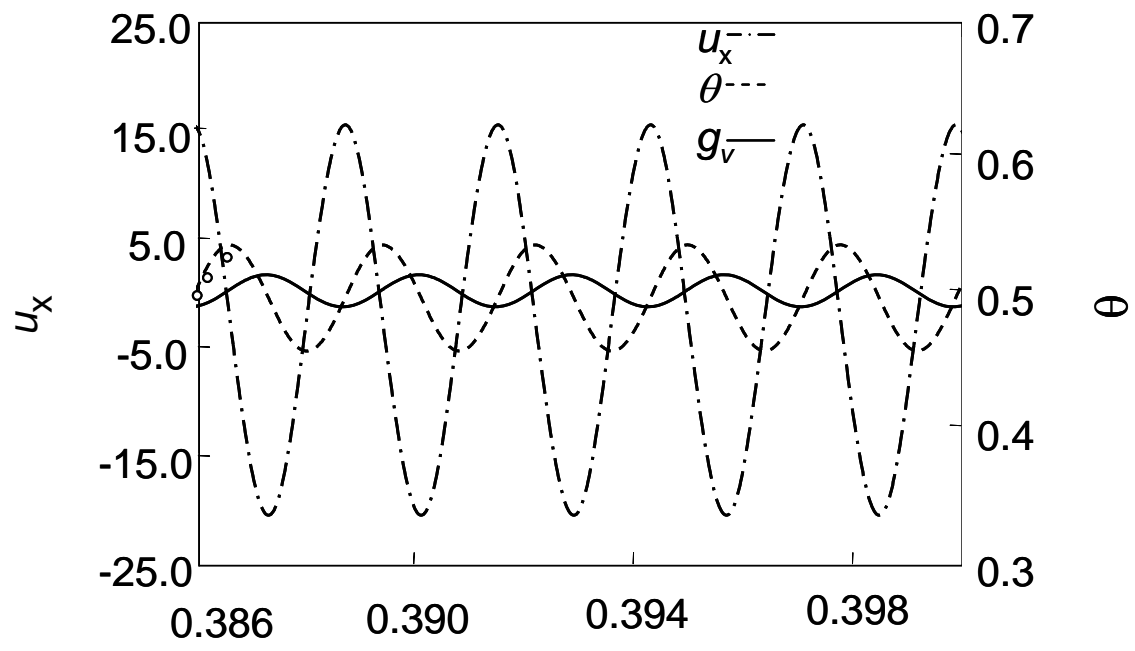

Fig. 3 Velocity $\left(u_{x}\right)$, temperature $(\theta)$ and vibrational acceleration $\left(g_{v}\right)$ at node $(25,65) . R a v=5 \times 10^{3}, \Omega=500$.

Fig. 3 Vitesse $\left(u_{x}\right)$, température $(\theta)$ et accélération vibrationnelle $\left(g_{v}\right)$ au nœud $(25,65) . R a v=5 \times 10^{3}, \Omega=500$. 


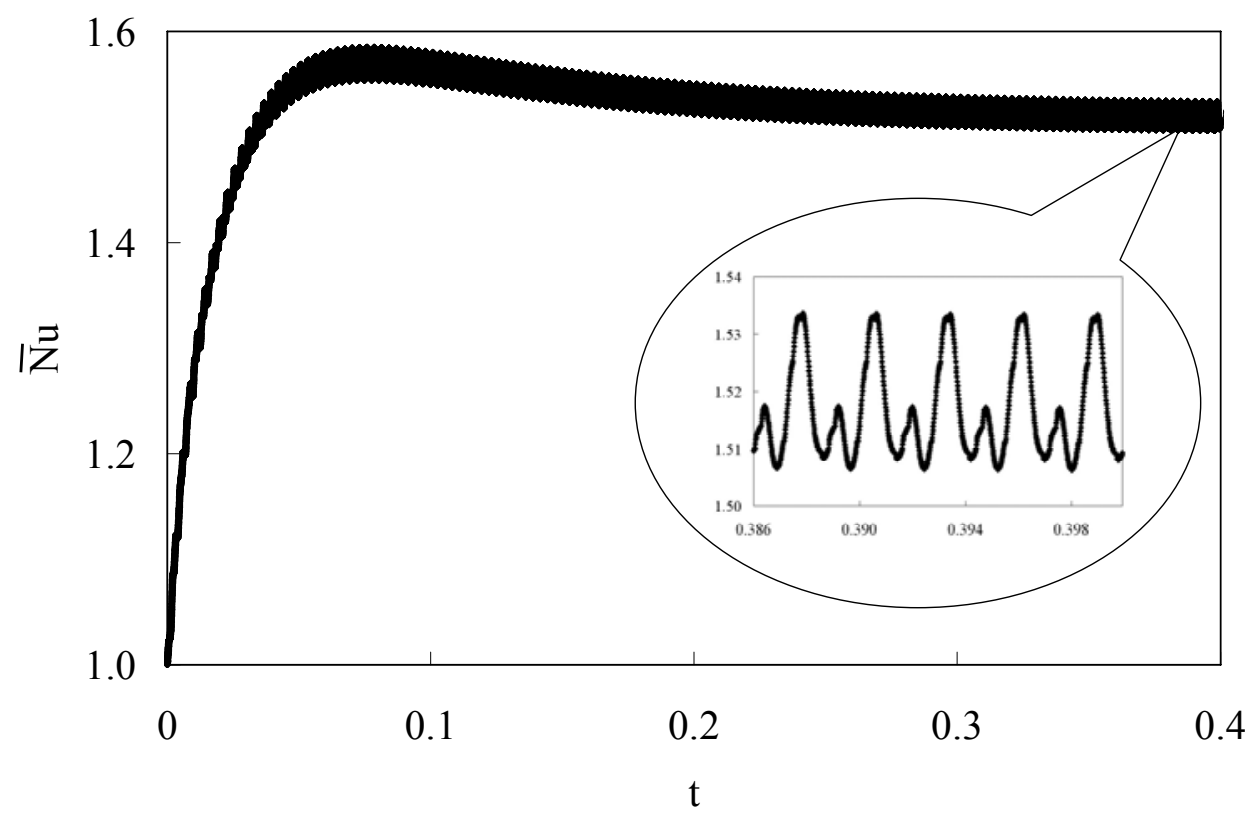

Fig. 4 Time history of the average Nusselt number at the cold vertical wall. Rav $=5 \times 10^{3}$, $\Omega=500$.

Fig. 4 Évolution du nombre de Nusselt à la paroi froide. $\operatorname{Rav}=5 \times 10^{3}, \Omega=500$. 


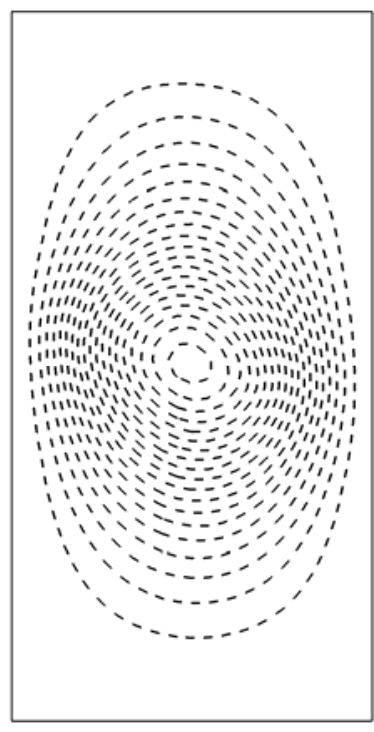

(a) $0 \pi$

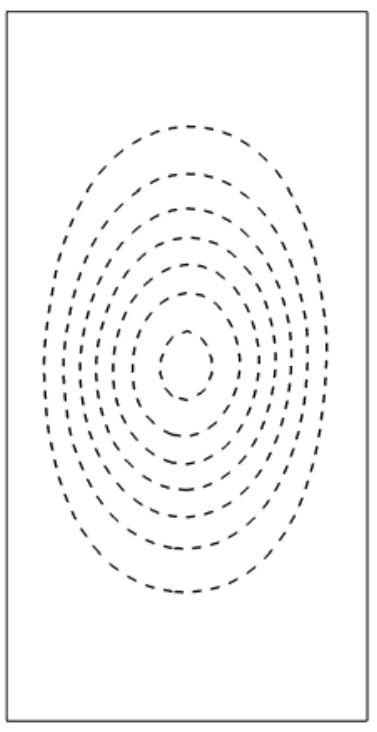

(b) $\frac{1}{2} \pi$

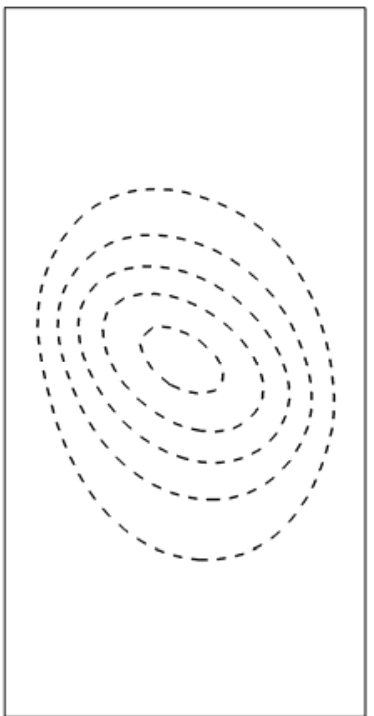

(f) $\frac{17}{10} \pi$

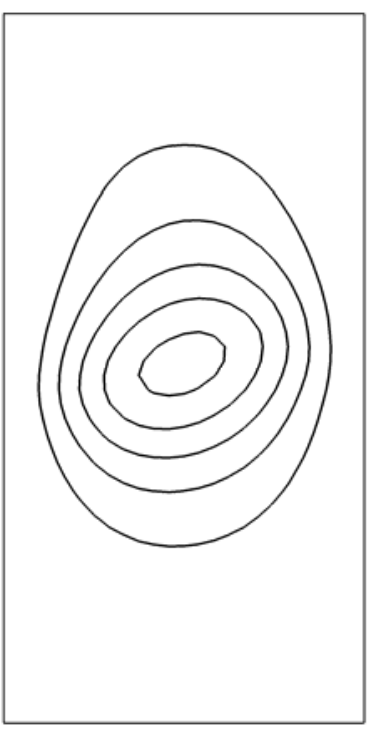

(c) $\frac{7}{10} \pi$

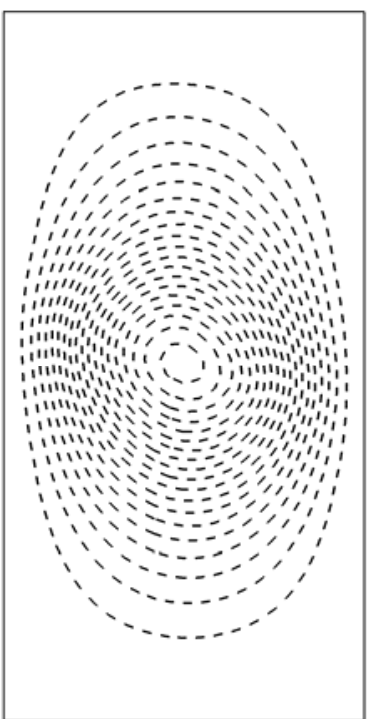

(g) $2 \pi$

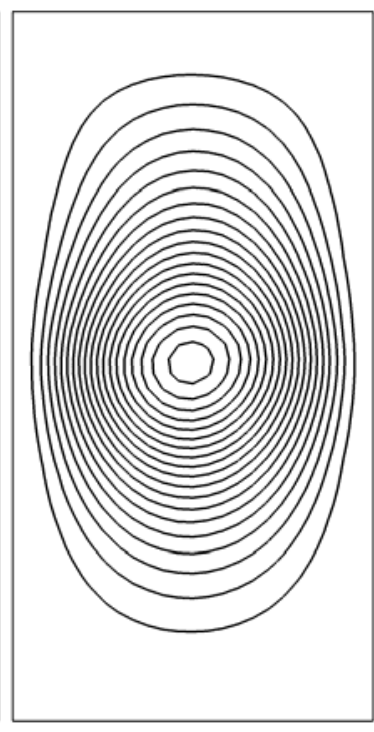

(d) $\pi$

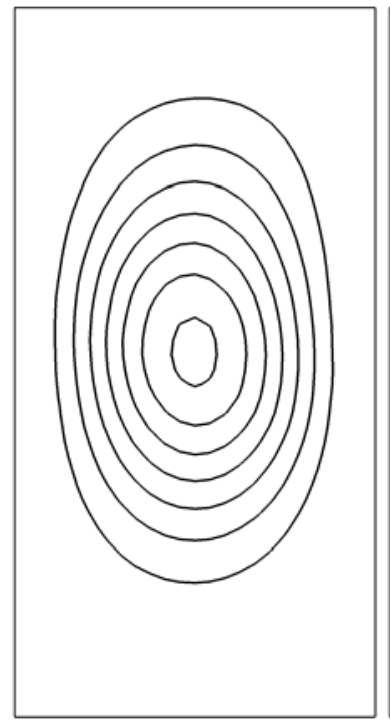

(e) $\frac{3}{2} \pi$

Fig. 5 Streamlines at different phases of one vibrational cycle after a steady oscillation is established. $R a v=5 \times 10^{3}, \Omega=500$. 
Fig. 5 Lignes de courant à différentes phases d'un cycle vibrationnel après l'établissement d'un régime oscillatoire. Rav $=5 \times 10^{3}, \Omega=500$.

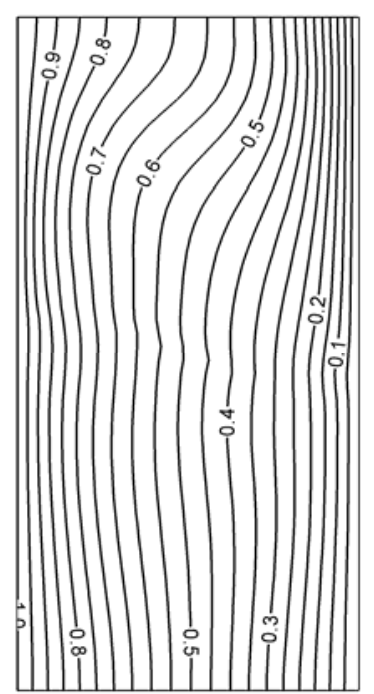

(a)

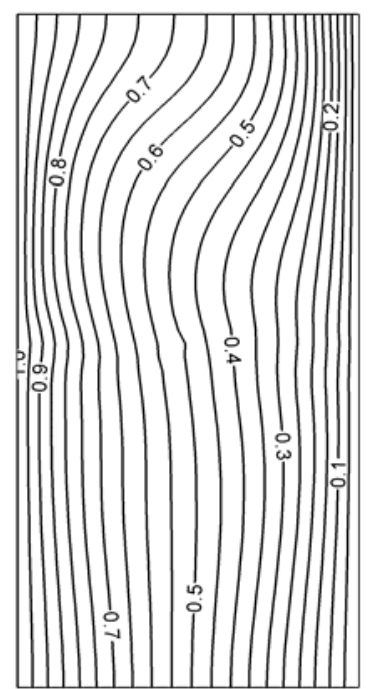

(b)

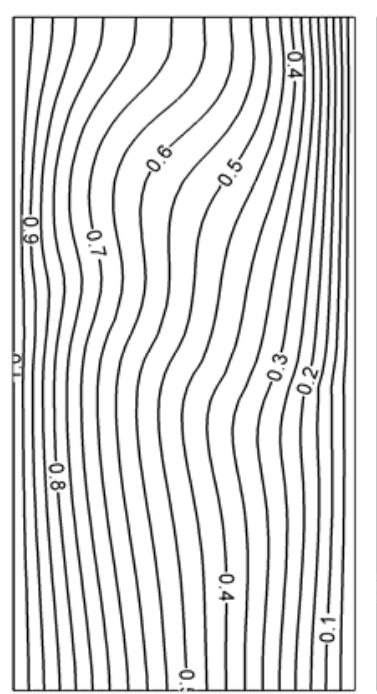

(c)

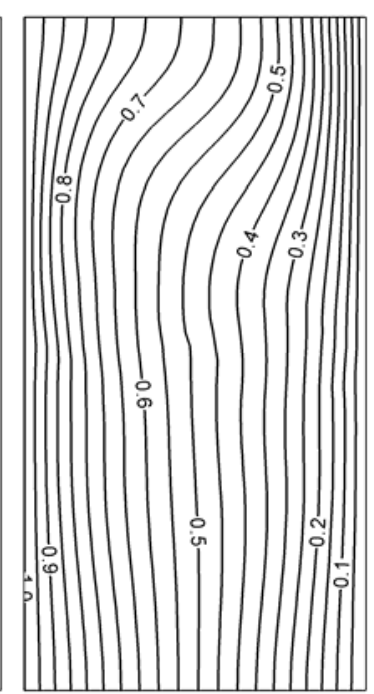

(d)

Fig. 6. Isotherms at three different phases of a cycle during a period after a steady oscillation is established. Rav $=5 \times 10^{3}, \Omega=500$. (a) 0 , (b) $\pi$, (c) $1.5 \pi$, (d) time averaged.

Fig. 6. Iothermes à trois phases d'un cycle durant une période après l'établissement d'un régime oscillatoire. $R a v=5 \times 10^{3}, \Omega=500$. (a) 0 , (b) $\pi$, (c) $1.5 \pi$, (d) moyenne temporelle. 


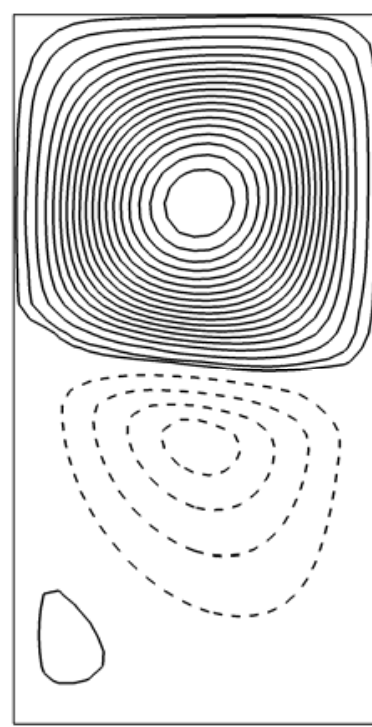

(a)

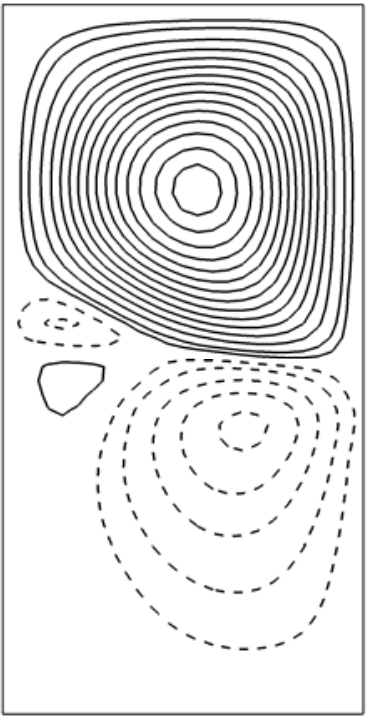

(b)

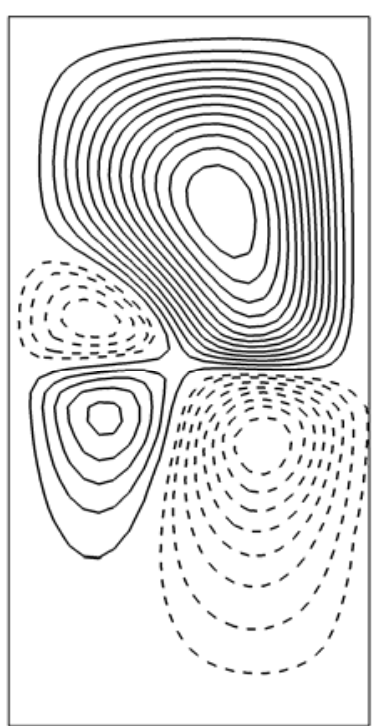

(c)

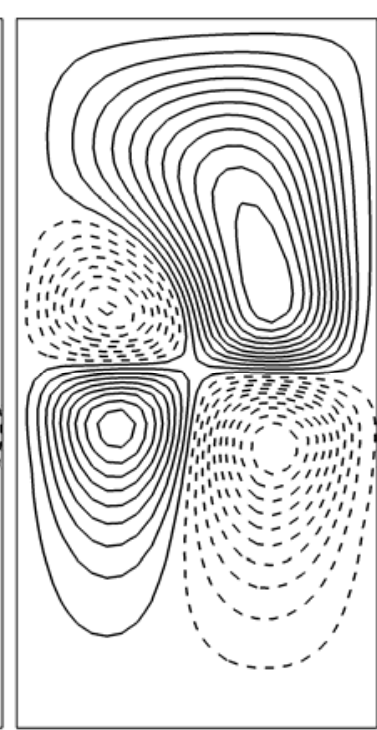

(d)

Fig. 7 Streamlines at $\Omega=500$ for different Rav. (a) 0, (b) 200 , (c) $10^{3}$, (d) $10^{4}$ Fig. 7 Lignes de courant $\Omega=500$ pour différents Rav . (a) 0 , (b) 200 , (c) $10^{3}$, (d) $10^{4}$ 


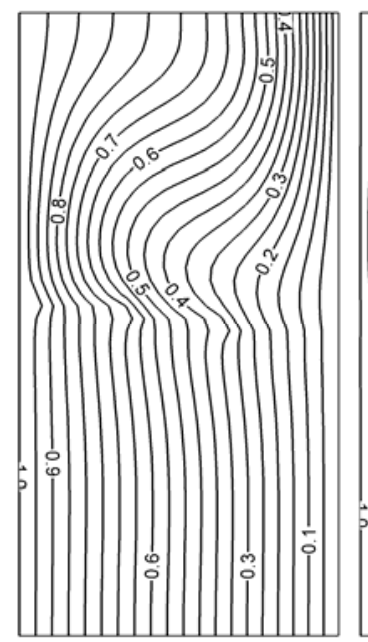

(a) $R a v=0$

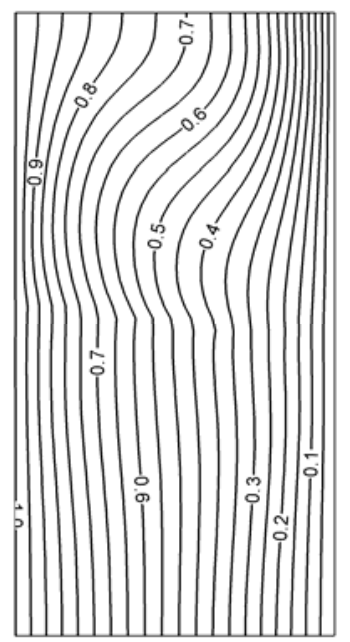

(b) $R a v=200$

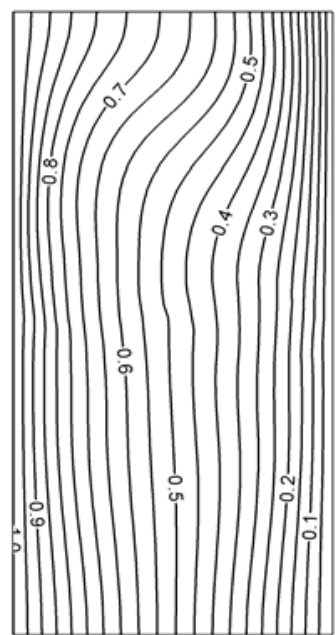

(c) $R a v=5 \times 10^{3}$

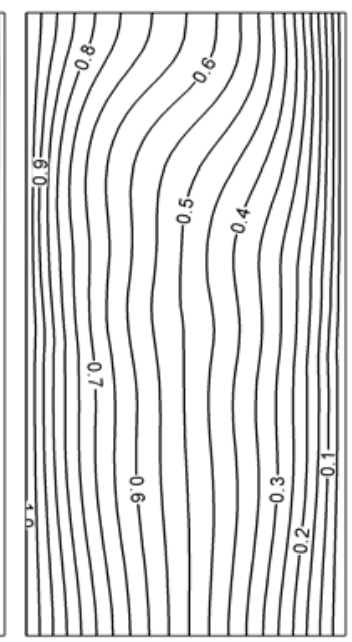

(d) $R a v=1 \times 10^{4}$

Fig.8 Time averaged isotherms at $\Omega=500$ for different Rav . Fig.8 Champ thermique moyen pour $\Omega=500$ et différents Rav . 


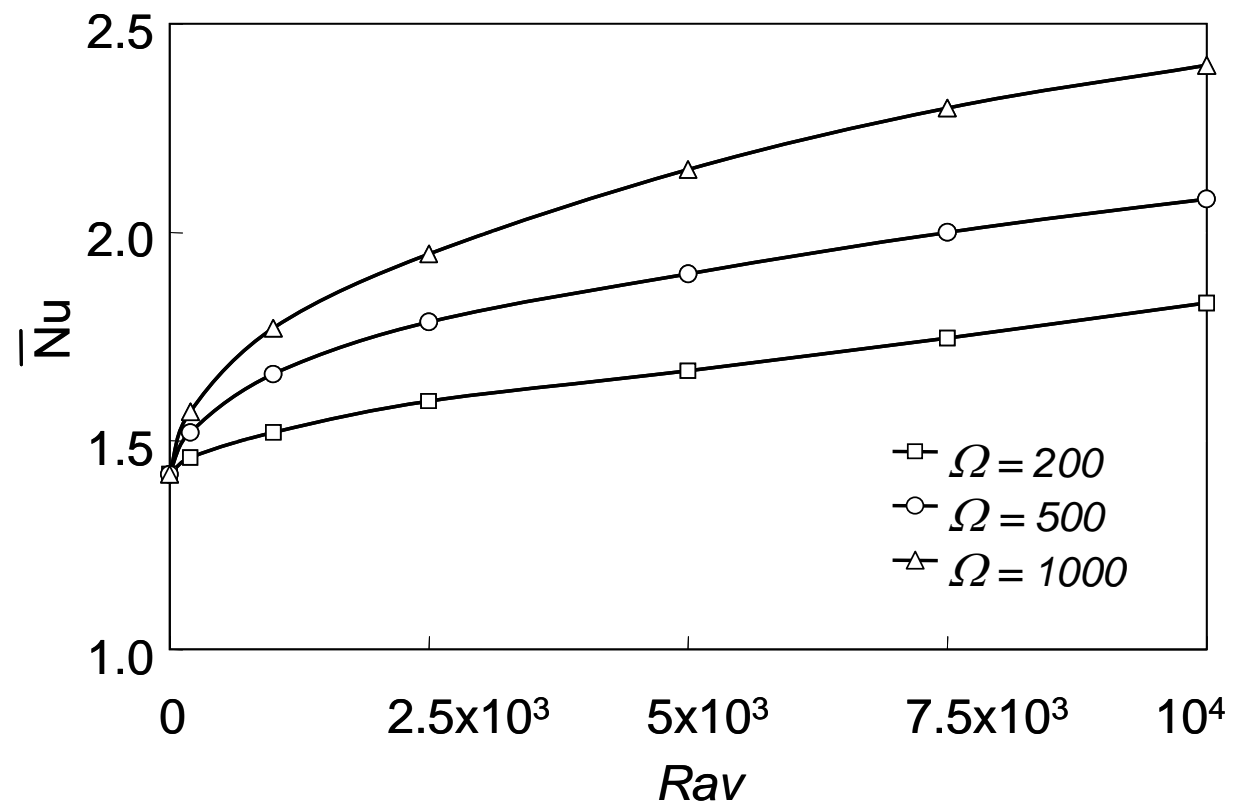

Fig. 9 Average Nusselt number at a vertical wall $\bar{N} u$ as a function of Rav for different frequencies $\Omega$.

Fig. 9 Nombre de Nusselt moyen $\bar{N} u$ à la paroi verticale fonction du Rav pour différentes fréquences $\Omega$. 


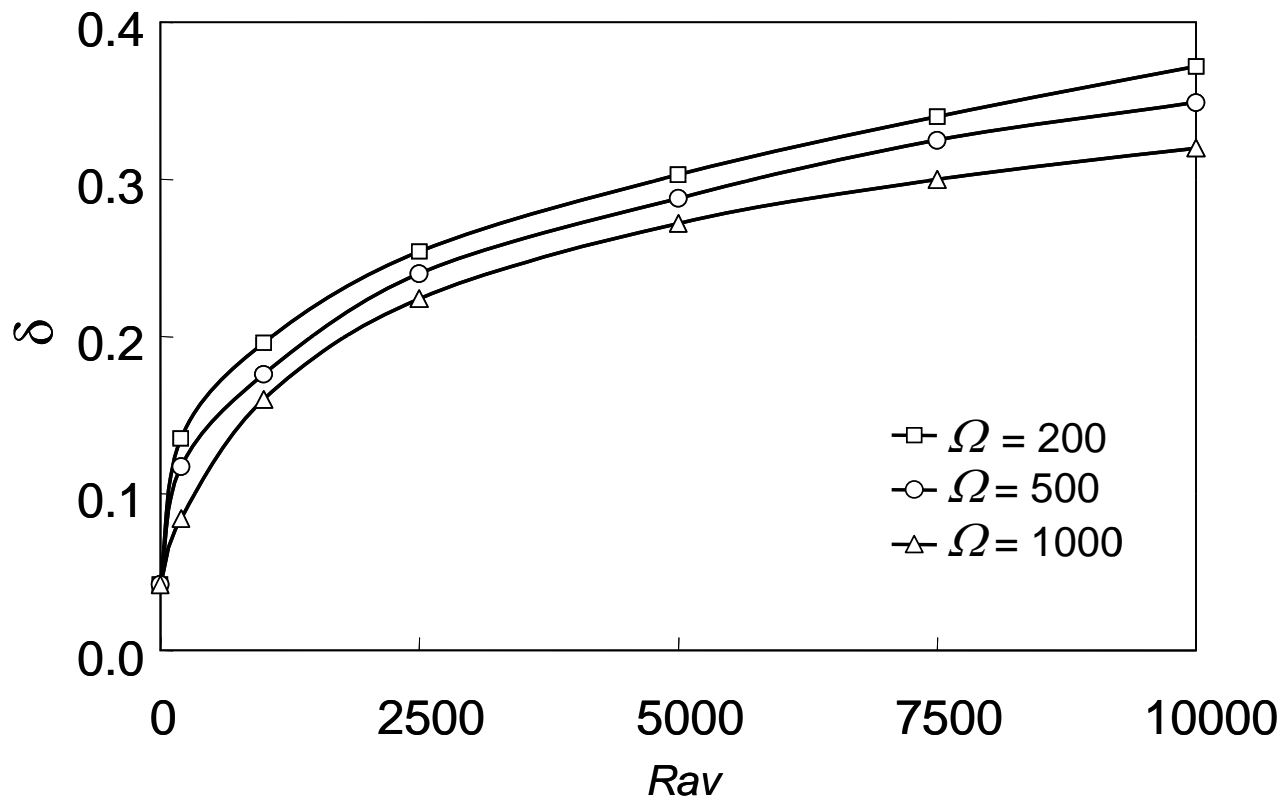

Fig. 10 Dimensionless interface fluctuation $\delta$ as a function of Rav for different frequencies $\Omega$.

Fig. 10 Fluctuation adimensionnelle $\delta$ de l'interface fonction de Rav pour différentes fréquences $\Omega$. 


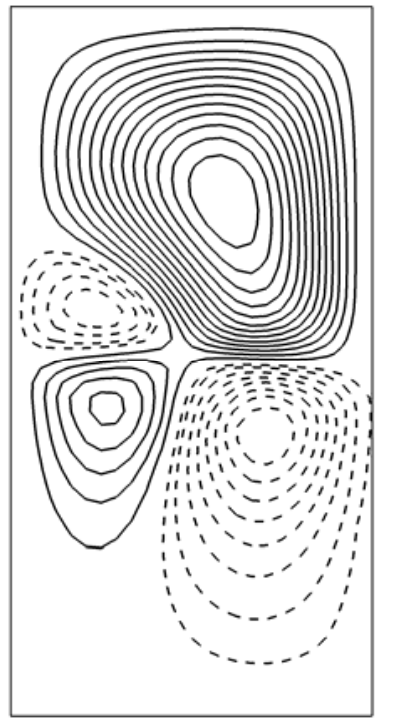

(a) thermal vibrational convection

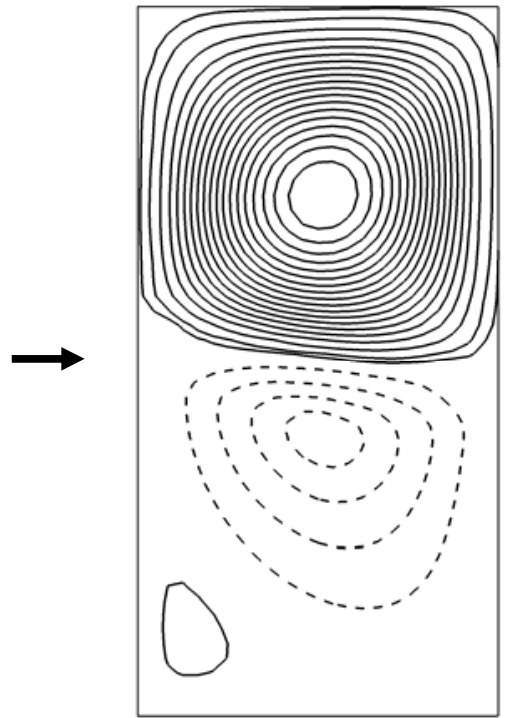

(b) steady gravity induced convection

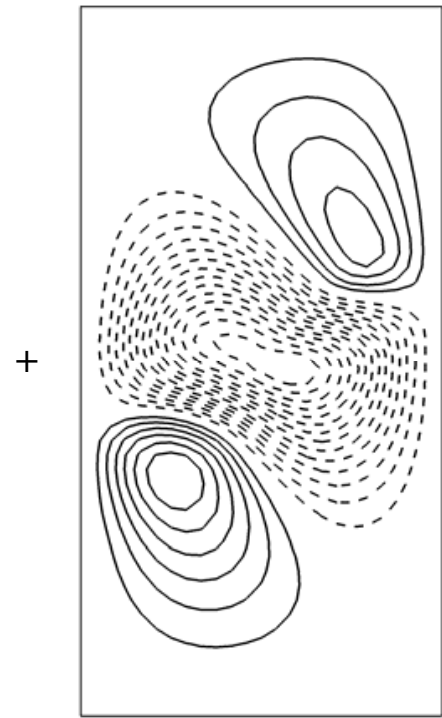

(c) vibration induced convection

Fig. 11 Decomposition of the thermal vibrational convection mechanism in the presence of a deformable interface between two immiscible liquids.

Fig. 11 Décomposition du mécanisme thermo-vibrationnel en présence d'interface déformable entre deux liquides immiscibles. 